\title{
Classification accuracy and consistency of computerized adaptive testing
}

\author{
Ying Cheng • Deanna L. Morgan
}

Published online: 6 September 2012

(C) Psychonomic Society, Inc. 2012

\begin{abstract}
In this article, four item selection methods in computerized adaptive testing are examined in terms of classification accuracy and consistency, including two popular heuristics for constraint management, the maximum priority index (MPI) method and the weighted deviation modeling method, as well as the widely known maximum Fisher information method and randomized item selection as baselines. Results suggest that the MPI method is able to meet constraints and keep test overlap rate low. Among the four methods, it is the only one that manages to produce parallel forms in terms of content coverage and, consequently, the only method to which the idea of classification consistency applies. With tests as short as 12 items, the MPI method does fairly well in classifying examinees accurately and consistently. Its performance improves with longer tests. The effects of number of decision categories and cut score locations are also examined. Recommendations are made in the Discussion section.
\end{abstract}

Keywords Computerized adaptive testing $\cdot$ Classification accuracy $\cdot$ Classification consistency $\cdot$ Constraint management $\cdot$ Exposure control $\cdot$ Test overlap rate

It has been argued that the ultimate purpose of testing is to make classification decisions (Cronbach \& Gleser, 1957; Rudner \& Guo, 2011), as evidenced by admission tests, certification tests, placement tests, graduation tests, and so on. In the context of computerized adaptive testing (CAT), discussion on classification accuracy has been more focused on algorithms that serve specifically classification purpose - that is, the computerized adaptive classification test (CACT) (e.g.,

\footnotetext{
Y. Cheng $(\bowtie)$

University of Notre Dame,

Notre Dame, IN, USA

e-mail: ycheng4@nd.edu

D. L. Morgan

The College Board,

Newtown, PA 18940, USA
}

Gnambs, \& Batinic, 2011; Eggen \& Straetmans, 2000; Rudner \& Guo, 2011; Weissman, 2007; Yang, Poggio, \& Glasnapp, 2006). In these studies, the test length is allowed to vary across examinees to reach confident decision for examinees through a sequential probability ratio test (Spray \& Reckase, 1994). For a comprehensive review of CACT, see Thompson (2007).

In reality, many adaptive testing programs are of fixed lengths and provide a score or a vector of scores that is used, either solely or together with other criteria, to make decisions on examinees. For example, the ACCUPLACER test, a test taken by millions of test takers around the world, is an adaptive test that is of fixed length and offers a final score based on ability estimate of item response theory (IRT). The ability estimate is compared against several cut thresholds as determined by the local institutions and may reflect as many as five levels of proficiency. The Graduate Management Admission Test, on the other hand, provides a vector of verbal, quantitative, and writing scores, which are adopted by business schools to make decisions on graduate student admission. So there are plenty of examples where regular, fixed-length CAT is used for classification purpose, yet discussion of classification accuracy (CA) for these CAT programs is sorely lacking in the literature.

Another important index for evaluating classification decision is classification consistency (CC), which has been discussed extensively in the context of linear test (e.g., Lee, 2010; Lee, Hanson, \& Brennan, 2002). In the context of adaptive testing, however, $\mathrm{CC}$ has not been examined at all, to our knowledge. $\mathrm{CC}$ is the degree of agreement between two independent administrations of the same test or parallel forms of the test (Kim, Choi, Um, \& Kim, 2006). Notably, psychometric parallelism, aside from equivalent statistical property of tests, also requires equivalent content coverage, word count, and key coverage (i.e., correct answers should fall approximately equally on each option for multiple-choice items) and so on. In adaptive testing, these are known as nonstatistical constraints (van der Linden, 2000). Special attention needs to be paid to the item 
selection algorithm to make sure that these constraints are met, since the most widely known item selection algorithm, the maximum Fisher information (MFI) method (Lord, 1980), is driven purely by asymptotic statistical optimality.

Another unique issue in adaptive testing is test overlap rate - that is, the average proportion of items that appear on two independently administered CAT. If two tests fully overlap, they would be perfectly parallel; but this seldom happens in CAT. In fact, too much test overlap in CAT may cause serious test security problems, since examinees may gain preknowledge on items that have appeared on previous examinees' tests (Wainer et al., 2000; Way, 1998).

In the CAT literature, test overlap rate has been linked to item exposure rate. According to Chen, Ankenmann, and Spray (2003),

$\bar{V}=\frac{\sum_{i=1}^{M} T_{i}\left(T_{i}-1\right)}{L \cdot N(N-1)}$,

where $\bar{V}$ is the expected test overlap rate, $T_{i}$ is the number of times the $i$ th item is exposed, $L$ is the test length, and $N$ is the number of examinees. This suggests that the more imbalanced the exposure rates are across the item pool, the higher the expected test overlap rate will be. It is therefore important to prevent items from being administered too frequently or overexposed (see Chang \& Ying, 1999; Davey \& Parshall, 1995; Lunz \& Stahl, 1998; Sympson \& Hetter, 1985).

There are many methods available to help combat item overexposure in CAT, such as the Sympson-Hetter approach (Sympson, \& Hetter, 1985), the $a$-stratification method and its variants (Chang, Qian, \& Ying, 2001; Chang \& Ying, 1999), the Kullback-Leibler information method and its variants (Barrada, Olea, Ponsoda, \& Abad, 2010; Chang \& Ying, 1996), the progressive method (Revuelta \& Ponsoda, 1998), and so on. For a review of item exposure control methods, please refer to Revuelta and Ponsoda (1998), Georgiadou, Triantafillou, and Economides (2007), and Barrada et al. (2010).

To discuss CC for CAT, an item selection algorithm needs to be able to produce adaptive tests that are content balanced and not unduly overlapping. Many constraint management methods for item selection have been proposed to balance measurement precision and constraint management (Moyer, Galindo, \& Dodd, 2012). They can largely be summarized into two categories. Some methods are based on mathematical programming, such as the "shadow test" method (van der Linden, 2002). At each step, a shadow test is formed that meets all the nonstatistical constraints with maximum information at the current ability estimate by using $0-1$ integer programming. The item in the shadow test with the maximum information is administered. The next shadow test needs to contain the previous shadow test, while meeting all the constraints. The mathematical programming methods, in general, handle constraint management very well. The drawback is that they are computationally intensive, especially when the number of constraints gets large. In the other category of methods, heuristics are used. These methods are, in general, computationally efficient. Their disadvantage is that the resulting tests are not guaranteed to meet all constraints at all times. Nonetheless, heuristics are widely used in operational testing programs due to their ease in implementation and computational efficiency. Item exposure control can be viewed as another nonstatistical constraint, meaning that the exposure rate of each item should be kept below a certain threshold.

By investigating the $\mathrm{CA}$ and $\mathrm{CC}$ of fixed-length CAT programs, this study fills in the gap in the current literature. Two popular heuristics for handling multiple constraints, the weighted deviation modeling (WDM) method (Stocking \& Swanson, 1993) and the maximum priority index (MPI) method (Cheng \& Chang, 2009; Cheng, Chang, Douglas, $\&$ Guo, 2009), are included. Both are able to incorporate exposure control as one of the constraints (Cheng \& Chang, 2009). They have been shown successful in previous studies in meeting multiple constraints, including exposure control. Therefore, they are capable of producing psychometrically parallel, but not excessively overlapping tests. The MFI method, the statistically optimal method, and the randomized item selection (RIS) method, a method that is least desirable statistically among the four but can produce the most balanced exposure rate in the item pool, are also included as baselines.

The rest of the article is organized as follows. First, we will review the four item selection methods included in the study. Next, we will delineate how to compute CA and CC indices in CAT. Results are then presented in the order of measurement precision, CA, and lastly, CC. Test length and the number of decision categories and cut score locations are varied. Finally, implications of the findings will be discussed.

\section{The item selection methods}

CAT is built upon IRT models, often the dichotomous ones. Equation 2 gives the widely used three-parameter logistic (3PL) model, which defines the probability of an examinee with ability $\theta$ giving a correct answer to an item as follows (Hambleton \& Swaminathan, 1985):

$$
\operatorname{Prob}\left(Y_{j}=1 \mid \theta\right)=c_{j}+\left(1-c_{j}\right)\left\{1+\exp \left[-a_{j}\left(\theta-b_{j}\right)\right]\right\}^{-1},
$$

where $Y_{j}=1$ represents a correct answer to item $j$ and $Y_{j}=0$ an incorrect one. $a_{j}, b_{j}$ and $c_{j}$ are, respectively, the discrimination, difficulty, and pseudo-guessing parameter values of the $j$ th item. 
One of the best known item selection methods in CAT is the MFI method (Lord, 1980). It selects the next item that maximizes the Fisher information evaluated at the current ability estimate point. Suppose $k$ items have been administered to an examinee and his or her current ability estimate is $\widehat{\theta}^{(k)}$. On the basis of the 3PL model, the Fisher information of the $j$ th item in the pool evaluated at $\widehat{\theta}^{(k)}$ is given by

$I_{j}\left(\widehat{\theta}^{(k)}\right)=\frac{\left(1-c_{j}\right) a_{i}^{2} \exp \left[-a_{j}\left(\widehat{\theta}^{(k)}-b_{j}\right)\right]}{\left\{1+\exp \left[-a_{j}\left(\widehat{\theta}^{(k)}-b_{j}\right)\right]\right\}^{2}\left\{1-c_{j}+c_{j}\left(1+\exp \left[-a_{j}\left(\widehat{\theta}^{(k)}-b_{j}\right)\right]\right)\right\}}$.

Then the item with the largest Fisher information at $\widehat{\theta}^{(k)}$ will be selected as the $(k+1)$ th item for the examinee.

Note that as $k$ approaches $\infty, \widehat{\theta}^{(k)} \rightarrow \theta$, and $\operatorname{se}\left(\widehat{\theta}^{(k)}\right) \rightarrow$ $\sqrt{1 / I(\theta)}$, where $\operatorname{se}\left(\widehat{\theta}^{(k)}\right)$ is the standard error of $\widehat{\theta}^{(k)}$ and $I$ $(\theta)$ represents the test information evaluated at $\theta$. Since $\theta$ is unknown, $I(\theta)$ is often approximated by $I\left(\widehat{\theta}^{(k)}\right)$. Assuming local independence, the test information is the sum of the Fisher information of the $k$ administered items. Therefore, by maximizing the item information at each turn, the MFI approach implies minimizing the standard error of $\widehat{\theta}$. It therefore optimizes measurement precision.

However, the MFI method does not take into account any nonstatistical constraints. The resulting tests may not follow the test blueprint; for example, they may not be content balanced. Furthermore, the MFI method tends to favor items with high discrimination parameters $(a)$ because of the $a^{2}$ term in information (see Eq. 3; Chang \& Ying, 1999; Chang et al., 2001). Therefore, the MFI method often results in very unbalanced exposure rates across the pool.

On the contrary, the RIS method picks items randomly from the pool. Asymptotically, it equalizes their exposure rates; hence, it approaches the best item pool utilization. But it takes into account neither items' statistical properties nor the nonstatistical constraints. Therefore, the resulting tests may severely violate the test specifications and may not be able to measure examinees' abilities accurately.

The MPI method and the WDM method, on the other hand, are methods developed for the purpose of managing multiple nonstatistical constraints. They therefore open up the possibility of presenting items meeting the test specifications better than do the MFI and the RIS methods. If exposure control is modeled as one constraint, they will also lead to more balanced item pool usage than will the MFI method. The price to pay for these gains is a compromise in measurement precision, although probably not as big a compromise as the RIS method results in.

The maximum priority index method can be considered as a variant of the maximum information method. Basically, it puts a multiplier in front of an item's Fisher information, and the product, instead of the information itself, will be maximized. The multiplier itself is a product of all the scaled "quota left" from the constraints. For example, assume that the item selection algorithm is evaluating an item measuring content area $m . T_{m}$ represents the number of items needed from this content area, and so far as $t_{m}$ items have been selected from it, then the scaled quota left is $\left(T_{m}-t_{m}\right) / T_{m}$. The larger the scaled quota left, the more urgent it is to fill in this gap, and the higher priority should be given to the item that is able to fulfill the constraint.

Meanwhile, the Fisher information measures the value the item has toward estimating $\theta$. The priority index, a product of the scaled quota left and the Fisher information, is therefore a measure of the overall "appeal" of the item in the constrained item selection process. The larger the product, the higher priority the item should be given. This method can be adapted to handle constraints that involve upper and lower bounds. It was shown to be very effective in managing multiple constraints simultaneously without losing much measurement precision (Cheng \& Chang, 2009).

The weighted deviation modeling method is a welldiscussed heuristic for constrained item selection in CAT (Buyske, 2005; Leung, Chang, \& Hau, 2005; Stocking \& Swanson, 1993). The objective function is a weighted sum of the deviations from constraints and the distance of the current test information from the target information value. For instance, if content area $m$ is underrepresented by $d_{m}$ items, the objective function could be $\sum_{m} w_{m} d_{m}+w_{I} d_{I}$, where $w_{m}$ is the weight associated with constraint $m, w_{I}$ is the weight on test information, and $d_{I}$ is the deficit from target test information. The next item is selected to minimize the objective function. Note that similar to the MPI method, the WDM method tries to find a balance between meeting constraints and optimizing measurement precision.

\section{Methodology}

CA refers to the extent to which the actual classifications using given cutoff scores agree with the "true" classifications. 
$\mathrm{CC}$ is defined as the extent to which the classifications agree with two independent administrations of the same form on the same test takers or of two parallel forms of the test (Lee et al., 2002)

Evaluating $\mathrm{CA}$ and $\mathrm{CC}$ requires cut scores. In this study, a national college placement CAT of 12 items is considered, and examinees are placed into five categories, from most basic competency level to most advanced. The four prespecified cut scores are $-0.0772,0.17523,0.45045$, and 1.0031 on the $\theta$ scale $\sim \mathcal{N}(0,1)$. In most operational CAT programs, the $\widehat{\theta}_{\mathrm{s}}$ are confined to a certain interval, so that nobody will receive a $\theta$ score of $+\infty$ or $-\infty$. For instance, some set the lowest $\widehat{\theta}$ to be -4.0 and the highest +4.0 . These are the bounds adopted in this study as well. Let's denote the lowest and highest value of $\widehat{\theta}$ as $c_{0}$ and $c_{H}$, where $H$ is the total number of categories. In this case, $H=5$. We now have an $(H+1)$ vector consisting of the two bounds and the cut scores $\left(\mathrm{c}_{0}, \mathrm{c}_{1}, \mathrm{c}_{2}, \mathrm{c}_{3}, \mathrm{c}_{4}, \mathrm{c}_{5}\right)$, where $c_{1}=-0.0772, c_{2}=0.17523, c_{3}=0.45045, c_{4}=1.0031$, and $c_{0}$ and $c_{5}$ are the two bounds - that is, -4.0 and 4.0, respectively. When an examinee's $\widehat{\theta}$ falls between $c_{h-1}$ and $c_{h}$, he or she will be classified into the $h$ th category.

Two stimulation studies are carried out to evaluate CA and $\mathrm{CC}$, respectively. Commonly across the two studies, the test length is 12, and a pool of 642 items is used.

\section{Simulation study 1}

Five thousand examinees are generated from $\sim \mathcal{N}(0,1)$. The $5,000 \theta \mathrm{s}$ generated are considered true $\theta \mathrm{s}$. The examinees can thus be classified to five categories. These are the true categories they belong to. Then four CATs are simulated, each featuring one item selection method. At the end, we obtain four sets of $\widehat{\theta}$ s. On the basis of each set of $\widehat{\theta}$, examinees can again be classified. These are their estimated categories.

A $5 \times 5$ contingency table can be constructed by comparing the true categorization with each of the four sets of categorizations based on $\widehat{\theta}_{\mathrm{s}}$. This process is replicated 100 times. A final contingency table can be obtained for each item selection method by averaging across the 100 replications. Each entry gives the proportion or relative frequency of examinees classified into corresponding categories. In the end, four contingency tables or matrices are produced. Note that each entry on the diagonal represents a joint probability - that is, the joint probability of truly belonging to a certain category and being thus classified. Hence, summing across the diagonal gives the proportions of examinees in the population that are correctly classified. The trace of each matrix is therefore the CA of an item selection method, denoted by $\gamma$. The sum of the upper diagonal entries indicates the probability of overestimating the competency level of examinees (denoted by $P^{+}$), while the sum of lower diagonal entries is the probability of underestimating the competency level (denoted by $P^{-}$).

\section{Simulation study 2}

$\mathrm{CC}$ is defined as the extent to which the classifications agree with two independent administrations of the same form on the same test takers or of two parallel forms of the test. Five thousand examinees are generated in the same way as above and are given two rounds of four adaptive tests. Four sets of $\widehat{\theta}$ are obtained in each round.

Four $5 \times 5$ contingency tables can thus be constructed on the basis of the $\widehat{\theta}$ s obtained from the two separate rounds of CAT administrations. Again, the process is replicated 100 times, and averages are taken to form the final contingency table for CC. The traces of the four matrices measure the agreement between the two independent administrations (denoted by $\lambda$ ). Note that this is the "raw" consistency measure, because it is not corrected for chance. Coefficient kappa $(\kappa)$ can be used to measure the consistency after chance correction (Cohen, 1960):

$\kappa_{h}=\frac{\operatorname{Pr}\left(g_{h}\right)-\operatorname{Pr}\left(\varepsilon_{h}\right)}{1-\operatorname{Pr}\left(\varepsilon_{h}\right)}$.

Here, $\operatorname{Pr}\left(g_{h}\right)$ is the observed proportion of cases that are classified into the same category $h$ both times, and $\operatorname{Pr}\left(\varepsilon_{h}\right)$ is the probability that such agreement is due to chance. In our study, the two administrations are independent. Therefore,

$\operatorname{Pr}\left(\varepsilon_{h}\right)=\left(p_{h}\right)^{2}$,

where $p_{h}$ is the expected proportion of simulees falling into the category $h$ under the underlying distribution of the test taker population. Therefore, $p_{h}$ can be computed by

$p_{h}=\int_{c_{h-1}}^{c_{h}} f(\theta) d_{\theta}$,

where $f(\theta)$ is the underlying distribution of the test taker population, and here it is $\mathcal{N}(0,1)$. And $c_{h^{-} 1}$ and $c_{h}$ are the two defining cutoffs for category $h$.

Given the cutoffs, we can easily obtain $p_{1}=0.47, p_{2}=0.10$, $p_{3}=0.10, p_{4}=0.17$, and $p_{5}=0.16$. Consequently, $\operatorname{Pr}\left(\varepsilon_{1}\right)=$ $0.22, \operatorname{Pr}\left(\varepsilon_{2}\right)=0.01, \operatorname{Pr}\left(\varepsilon_{3}\right)=0.01, \operatorname{Pr}\left(\varepsilon_{4}\right)=0.03$, and $\operatorname{Pr}\left(\varepsilon_{5}\right)=0.03$. Note that in reality, the theoretical distribution of $f(\theta)$ is unknown and has to be estimated from data.

\section{Results}

For most testing programs, even though the scores are eventually used to facilitate a classification decision, providing an accurate latent trait estimate is still very important. So let's first examine how well the latent trait is recovered. 
Three overall measurement precision indices are calculated: overall bias, mean square error (MSE), and the Pearson correlation between $\theta$ and its estimate $\widehat{\theta}$ :

Bias $=\frac{\sum_{i=1}^{N}\left(\widehat{\theta}_{i}-\theta_{i}\right)}{N}$

$M S E=\frac{\sum_{i=1}^{N}\left(\widehat{\theta}_{i}-\theta_{i}\right)^{2}}{N}$

$\rho_{\hat{\theta, \hat{\theta}}}=\frac{\sum_{i=1}^{N}\left(\theta_{i}-\bar{\theta}\right)\left(\widehat{\theta}_{i}-\widehat{\theta}\right)}{S_{\theta} S_{\widehat{\theta}}}$,

where $N$ is the number of examinees, $\theta_{\mathrm{i}}$ and $\widehat{\theta}_{i}$ are the estimated and true abilities of the $i$ th examinee, and $S_{\theta}$ and $S_{-}$ are the standard deviations of the true and estimated abilities, respectively. The results are summarized in Table 1. Note that the values are averages of 100 replications from study 1 .

As was expected, all four item selection methods lead to essentially unbiased results. This is basically a property of maximum likelihood estimate. Table 1 , however, clearly indicates that the MFI method is the superior, followed by both MPI and WDM, and RIS is a distant last in terms of the magnitude of MSE. This result turns out to have strong implications for the results of $\mathrm{CA}$ and $\mathrm{CC}$, which will be discussed in detail in the next sections. The correlation between true latent trait and its estimate also suggests the same rank ordering. Notably, the two constraint management methods, the MPI method and the WDM method, manage to maintain a high level of $\rho_{\theta, \hat{\theta}}$. This is in stark contrast with their relative size of MSE (as compared with that of the MFI method), although the absolute magnitude of their MSE is not alarming. Between MPI and WDM, the difference is inconsequential.

\section{Classification accuracy}

Four CA tables are generated, one for each item selection method (see Tables 2, 3, 4, and 5). Each table can also be considered a matrix and is denoted by the item selection method it features. For example, Table 2 is matrix MFI.

Table 1 Overall measurement precision indices

\begin{tabular}{lccc}
\hline Methods & Bias & MSE & $\rho_{\theta, \widehat{\theta}}$ \\
\hline MFI & 0.01 & 0.09 & 0.96 \\
MPI & 0.01 & 0.16 & 0.93 \\
WDM & 0.02 & 0.17 & 0.93 \\
RIS & -0.06 & 1.14 & 0.79 \\
\hline
\end{tabular}

Table 2 Classification accuracy by using the maximum Fisher information method

\begin{tabular}{llllll}
\hline & 1 & 2 & 3 & 4 & 5 \\
\hline 1 & .440 & 0.032 & 0.002 & 0.001 & 0.000 \\
2 & .026 & 0.051 & 0.024 & 0.003 & 0.000 \\
3 & .002 & 0.019 & 0.050 & 0.024 & 0.000 \\
4 & .000 & 0.001 & 0.016 & 0.124 & 0.022 \\
5 & .000 & 0.000 & 0.000 & 0.019 & 0.142 \\
$\gamma=.81$ & & $P^{+}=.11$ & & $P^{-}=.08$ & \\
\hline
\end{tabular}

Note that in each table, the rows represent the "true" categories, and the columns represent the "estimated" categories. For instance, the entry $\mathrm{MFI}_{1,2}$ (i.e., the first row and the second column of Table 2 ) is . 03 , meaning that about $3 \%$ of the examinees in the population who really belong to category 1 are classified to category 2 .

The trace of each matrix is the CA rate, the sum of the upper triangle, $P^{+}$, is the overclassification rate, and the sum of the lower triangle, $P^{-}$, is the underclassification rate. The sum of $P^{+}$and $P^{-}$is the misclassification rate, and it equals (1-trace). Not surprisingly, the MFI method yields the highest CA rate, $81 \%$, as well as the lowest $P^{+}$and the lowest $P^{-}$. The RIS method is the opposite: It leads to the smallest trace and the largest $P^{+}$and $P^{-}$. The two constraint management methods fall in between.

What is more interesting is how WDM and MPI compare against each other. Results from Tables 3 and 4 suggest that these two methods lead to really similar CA rates, as well as $P^{+} \mathrm{s}$ and $P^{-} \mathrm{s}$. This is consistent with the observations from the overall measurement precision results of the two methods.

Recall that the bias from the MFI, WDM, and MPI methods are all similar and negligible. So the loss of CA in the WDM and MPI methods (as compared against the MFI method) is caused by larger variability (i.e., standard error) of their latent trait estimates. This is consistent with Rudner (2005), which explicitly formulated the standard error of maximum likelihood estimate of the latent trait as part of the computation of CA.

Table 3 Classification accuracy by using the maximum priority index method

\begin{tabular}{llllll}
\hline & 1 & 2 & 3 & 4 & 5 \\
\hline 1 & .418 & 0.040 & 0.015 & 0.004 & 0.000 \\
2 & .028 & 0.032 & 0.030 & 0.012 & 0.001 \\
3 & .007 & 0.019 & 0.038 & 0.031 & 0.001 \\
4 & .001 & 0.006 & 0.024 & 0.105 & 0.028 \\
5 & .000 & 0.000 & 0.000 & 0.018 & 0.143 \\
$\gamma=.74$ & & $P^{+}=.16$ & & $P^{-}=.10$ & \\
\hline
\end{tabular}


Table 4 Classification accuracy by using the weighted deviation modeling method

\begin{tabular}{llllll}
\hline & 1 & 2 & 3 & 4 & 5 \\
\hline 1 & .423 & 0.037 & 0.013 & 0.003 & 0.000 \\
2 & .028 & 0.038 & 0.028 & 0.010 & 0.000 \\
3 & .007 & 0.022 & 0.039 & 0.027 & 0.001 \\
4 & .000 & 0.004 & 0.021 & 0.108 & 0.030 \\
5 & .000 & 0.000 & 0.000 & 0.020 & 0.141 \\
$\gamma=.75$ & & $P^{+}=.15$ & & $P^{-}=.10$ & \\
\hline
\end{tabular}

\section{Classification consistency}

Before we summarize the CC tables, it is important to reiterate here that the definition of $\mathrm{CC}$ is based on the notion of parallel forms. In a CAT scenario, it is clear that even the same examinee might receive different items during two administrations. By tailoring to each examinee's ability level, the two adaptive tests an examinee receives are presumably parallel in terms of test information. However, without proper constraint management techniques, the two adaptive tests will not be parallel in the test development sense.

In this study, in total, 21 constraints are involved. If exposure control is viewed as a constraint, there are, in total, 22 constraints: 15 of them are for content-balancing purposes. Other constraints concern item type and key distribution. The content areas are very fine grained. For example, one content constraint requires that 1 to 2 items should be on solving linear equations and inequalities, given that the test length is 12 . An example of the item type constraint specifies that at most 2 items can have negative stems. Key distribution constraint requires that at least 2 items and at most 5 items have one of the four options, A, B, C, or D, as the answer key (see Table 6 for details of the constraints). In Table 6, the constraints are expressed in percentages. For example, constraint 18 states that from $17 \%$ to $42 \%$ of the items should have a certain characteristic - for example, a content area, or an item type, and so forth. Given a 12-item test, that means

Table 5 Classification accuracy by using the randomized item selection method

\begin{tabular}{llllll}
\hline & 1 & 2 & 3 & 4 & 5 \\
\hline 1 & .404 & 0.043 & 0.019 & 0.008 & 0.002 \\
2 & .036 & 0.027 & 0.022 & 0.017 & 0.002 \\
3 & .015 & 0.020 & 0.023 & 0.028 & 0.009 \\
4 & .006 & 0.011 & 0.028 & 0.071 & 0.048 \\
5 & .000 & 0.001 & 0.003 & 0.027 & 0.131 \\
$\gamma=.66$ & & $P^{+}=.20$ & & $P^{-}=.15$ & \\
\hline
\end{tabular}

Table 6 Constraint specification (\%)

\begin{tabular}{lcc}
\hline Constraint & Min & Max \\
\hline C1 & 0 & 8 \\
C2 & 8 & 8 \\
C3 & 0 & 8 \\
C4 & 0 & 8 \\
C5 & 8 & 8 \\
C6 & 8 & 8 \\
C7 & 8 & 8 \\
C8 & 0 & 8 \\
C9 & 8 & 8 \\
C10 & 8 & 8 \\
C11 & 8 & 17 \\
C12 & 8 \\
C13 & 0 & 8 \\
C14 & 0 & 8 \\
C15 & 8 & 8 \\
C16 & 0 & 8 \\
C17 & 0 & 17 \\
C18 & 0 & 42 \\
C19 & 0 & 42 \\
C20 & 17 & 42 \\
C21 & 17 & 42 \\
\hline
\end{tabular}

2-5 items should have this characteristic. Given a 20item test, that would mean $3-8$ items should have this characteristic.

Figure 1 shows the constraint management efficacy of each item selection method for the 12 -item test. The horizontal axis is the number of constraints that are violated, and the vertical axis shows the proportion of tests that violate a certain number of constraints. It is clear from Fig. 1 that the MPI method is the best among all four item selection

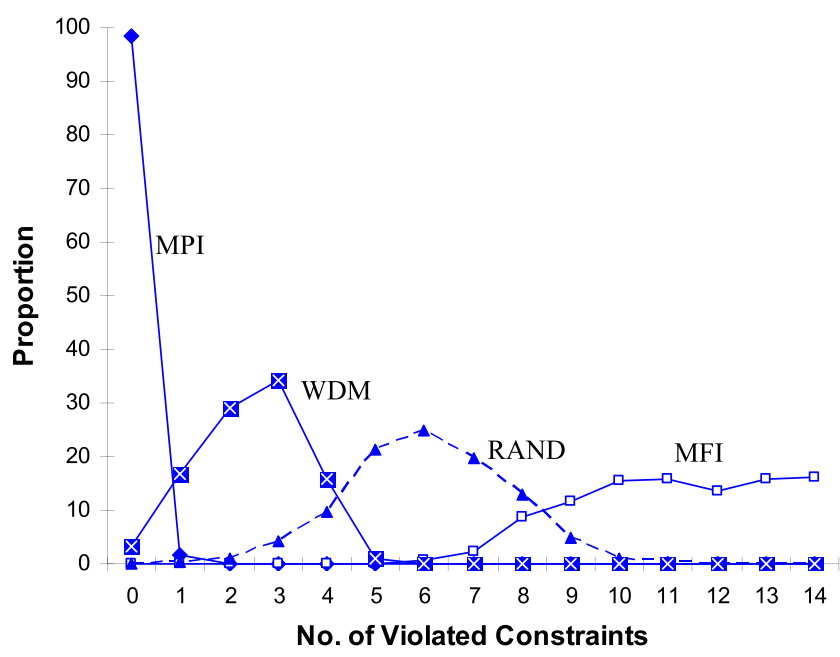

Fig. 1 Performance on constraint management of the four item selection methods 
Table 7 Classification consistency by using the maximum priority index method

\begin{tabular}{llllll}
\hline & 1 & 2 & 3 & 4 & \multicolumn{1}{l}{5} \\
\hline 1 & .391 & 0.043 & 0.022 & 0.008 & 0.000 \\
2 & .038 & 0.021 & 0.018 & 0.012 & 0.001 \\
3 & .019 & 0.022 & 0.029 & 0.031 & 0.003 \\
4 & .007 & 0.011 & 0.031 & 0.081 & 0.035 \\
5 & .001 & 0.001 & 0.002 & 0.035 & 0.136 \\
$\lambda=.66$ & & & Overlap rate $=.16$ &
\end{tabular}

methods in terms of constraint management. Nearly $100 \%$ of the tests by the MPI method are violation free. The WDM method manages to curb the constraint violation somewhat effectively, producing the majority of tests with fewer than three content violations. The other two methods lead to many constraint violations.

Strictly speaking, only the MPI is capable of producing parallel adaptive tests. Table 7 gives the $\mathrm{CC}$ rate by using the MPI method. Tables 8, 9, and 10 give the "pseudo"-CC rates for the other three item selection methods, respectively. The prefix "pseudo" emphasizes that when constraint management fails, resulting tests are not really parallel, and the concept of CC does not apply. Consequently, the numbers in Tables 8, 9, and 10 cannot be taken at their face values. Please use caution when interpreting these numbers.

The trace gives the average proportion of examinees that are classified into the same categories on both administrations. The test overlap rate is also reported. It is the average number of items in common on both administrations divided by the test length. The larger the test overlap rate, the more items are in common across administrations. As was discussed earlier, in operational CAT programs, it is not desirable to have a large test overlap rate, for test security reasons.

Table 7 shows that with the MPI method, the $\mathrm{CC}$ rate is .66. This seems a little low, and it has two implications. First, the test is only 12 items long. As is shown in Table 1, the MPI method with a 12-item test yields larger variability in ability estimate than the MFI method does. The variability finally enters $\mathrm{CC}$ as it does $\mathrm{CA}$. To improve both $\mathrm{CA}$ and

Table 8 Classification consistency by using the maximum Fisher information method

\begin{tabular}{llllll}
\hline & 1 & 2 & 3 & 4 & 5 \\
\hline 1 & .427 & 0.032 & 0.006 & 0.001 & 0.000 \\
2 & .033 & 0.037 & 0.022 & 0.008 & 0.000 \\
3 & .008 & 0.021 & 0.035 & 0.028 & 0.000 \\
4 & .001 & 0.010 & 0.030 & 0.105 & 0.029 \\
5 & .000 & 0.000 & 0.000 & 0.030 & 0.136 \\
$\lambda=.74$ & & & \multicolumn{5}{l}{ Overlap rate $=.61$} & \\
\hline
\end{tabular}

Table 9 Classification consistency by using the weighted deviation modeling method

\begin{tabular}{llllll}
\hline & 1 & 2 & 3 & 4 & 5 \\
\hline 1 & .396 & 0.034 & 0.021 & 0.004 & 0.000 \\
2 & .035 & 0.024 & 0.021 & 0.014 & 0.001 \\
3 & .019 & 0.025 & 0.034 & 0.031 & 0.002 \\
4 & .007 & 0.015 & 0.030 & 0.084 & 0.027 \\
5 & .000 & 0.000 & 0.002 & 0.036 & 0.138 \\
$\lambda=.68$ & & & \multicolumn{2}{l}{ Overlap rate $=.31$} &
\end{tabular}

$\mathrm{CC}$, the test probably needs to be lengthened. On the other hand, even the MFI method achieves only a CC rate at .74 . Taking that baseline into account, the MPI method is not doing that bad, especially when the test overlap rate for the MPI method is .16 (the maximum exposure rate is set at .2 for exposure control in the MPI method), as compared with .61 from the MFI method. As a side note, the WDM method leads to a CC rate of .67 , similar to the MPI method.

Also notably, the rate of being classified to the same category or \pm 1 category in two independent administrations is .91. This indicates that even though the chance of being classified into the same category is only about $60 \%$, most likely the test will place the examinee in either the same or an adjacent category.

After chance correction, the CC rate for the MPI method is .41. This means that the MPI method improves the CC rate from random guessing by $41 \%$. This again, seems not very impressive, but as was explained earlier, given the test length, the MPI method is doing well. Since this study examines a placement test that is generally deemed lowstake, the level of CA and CC may be fine. For a higherstake test, a longer test is probably warranted. To explore the effect of test length, we simulated two additional test lengths, 20 and 40 items.

\section{Effect of longer tests}

As was demonstrated above, the MPI method works fairly well when the test length is 12 . Intuitively, longer tests will

Table 10 Classification consistency by using the randomized item selection method

\begin{tabular}{llllll}
\hline & 1 & 2 & 3 & 4 & 5 \\
\hline 1 & .365 & 0.041 & 0.025 & 0.017 & 0.003 \\
2 & .044 & 0.016 & 0.017 & 0.015 & 0.005 \\
3 & .030 & 0.012 & 0.018 & 0.025 & 0.014 \\
4 & .018 & 0.017 & 0.023 & 0.053 & 0.048 \\
5 & .004 & 0.005 & 0.012 & 0.044 & 0.127 \\
$\lambda=.58$ & & & \multicolumn{5}{c}{ Overlap rate $=.03$} & \\
\hline
\end{tabular}


Table 11 Classification accuracy by using the MPI method, medium test length

\begin{tabular}{llllll}
\hline & 1 & 2 & 3 & 4 & 5 \\
\hline 1 & .439 & 0.036 & 0.008 & 0.000 & 0.000 \\
2 & .031 & 0.047 & 0.022 & 0.004 & 0.000 \\
3 & .005 & 0.020 & 0.047 & 0.022 & 0.000 \\
4 & .000 & 0.003 & 0.021 & 0.105 & 0.022 \\
5 & .000 & 0.000 & 0.000 & 0.019 & 0.148 \\
$\gamma=.79$ & & bias $=.001$ & & \\
MSE $=.088$ & & $\rho_{\theta, \theta}=.962$ & & \\
\hline
\end{tabular}

lead to better latent trait recovery and, in turn, better classification decisions. Tables $11,12,13$, and 14 show measurement precision statistics, $\mathrm{CA}$ and $\mathrm{CC}$ for test length equals 20 (40). Results are reported only for the MPI method because of its superior performance shown above. Comparing Table 7 against Table 11 and Table 1 we can tell that by increasing test length from 12 to $20, M S E$ is reduced by almost half for the MPI method, and CA increases from .74 to .79. Meanwhile, Table 12 shows that $\mathrm{CC}$ climbs from .66 to .71 . If the test length is further prolonged to 40 , measurement precision also continues to improve, although at a slower rate (see Table 13). CA and CC will reach .80 and .72 , respectively.

On the surface, the improvement on classification decision is minimal by adding more items. However, a decisiontheoretic perspective can help us to find out more about the advantages of longer tests. The $P^{+}$and $P^{-}$defined above do not consider the severity of different errors. For example, classifying an examinee who, in reality, belongs to the highest category to the second highest category is a less severe error than classifying him or her to the lowest category. Taking these concerns in consideration, we looked at the following indices: classifying too high by one category $\left(P_{1}^{+}\right)$, by two categories $\left(P_{2}^{+}\right)$, by three categories $\left(P_{3}^{+}\right)$, and by four categories $\left(P_{4}^{+}\right)$. Similarly, we can define $P_{1}^{-}$to $P_{4}^{-}$. To summarize the overall error rate, we can sum up these indices. Note that $P_{1}^{+}+P_{2}^{+}+P_{3}^{+}+P_{4}^{+}=P^{+}$and $P_{1}^{-}+P_{2}^{-}$ $+P_{3}^{-}+P_{4}^{-}=P^{-}$. These are unweighted error rates. If we

Table 12 Classification consistency by using the MPI method, medium test length

\begin{tabular}{llllll}
\hline & 1 & 2 & 3 & 4 & 5 \\
\hline 1 & .414 & 0.040 & 0.020 & 0.002 & 0.000 \\
2 & .040 & 0.030 & 0.027 & 0.009 & 0.000 \\
3 & .014 & 0.026 & 0.035 & 0.021 & 0.002 \\
4 & .002 & 0.005 & 0.025 & 0.093 & 0.025 \\
5 & .000 & 0.000 & 0.001 & 0.029 & 0.140 \\
$\lambda=.71$ & & & \multicolumn{4}{c}{ Chance corrected $=.47$} \\
\hline
\end{tabular}

Table 13 Classification accuracy by using the MPI method, long test

\begin{tabular}{|c|c|c|c|c|c|}
\hline & 1 & 2 & 3 & 4 & 5 \\
\hline 1 & .443 & 0.036 & 0.005 & 0.000 & 0.000 \\
\hline 2 & .026 & 0.051 & 0.023 & 0.005 & 0.000 \\
\hline 3 & .005 & 0.019 & 0.051 & 0.020 & 0.000 \\
\hline 4 & .000 & 0.002 & 0.023 & 0.104 & 0.022 \\
\hline 5 & .000 & 0.000 & 0.000 & 0.022 & 0.145 \\
\hline \multicolumn{3}{|c|}{$\gamma=0.80$} & \multicolumn{3}{|c|}{ bias $=0.008$} \\
\hline \multicolumn{3}{|c|}{$\mathrm{MSE}=.081$} & \multicolumn{3}{|c|}{$\rho_{\theta, \widehat{\theta}}=.964$} \\
\hline
\end{tabular}

apply heavier weights on more severe errors-for instance, 1 for $P_{1}^{+}$or $P_{1}^{-}, 5$ for $P_{2}^{+}$or $P_{2}^{-}, 10$ for $P_{3}^{+}$or $P_{3}^{-}$, and 20 for $P_{4}^{+}$ or $P_{4}^{-}$- the weighted sums offer weighted error rates. Table 15 provides the summary of these error rates for test length of 12, 20, and 40 . Clearly, with 20 items or more, no examinee is classified two categories above or below his or her true category. From 12 to 20 items, the weighted error rate is reduced from .48 to .29 , and it further drops to .27 when test length increases to 40 .

\section{Effect of the number of classification categories}

As was pointed out by one reviewer, "classification accuracy quickly gets unsatisfying if the number of categories grows." Results shown above concern classifying test takers into five performance categories - for example, remedial II, remedial I, proficient, advanced I, and advanced II. Suppose that, instead of the fine categorization, a cruder decision is being made - that is, pass or fail. Anyone in the proficient category and above will pass; otherwise, he or she will fail. Then the cut score that separates remedial I and proficient in the original five-category classification becomes the cut that defines pass or fail. That is, $c_{2}=$ .17523 , on p. 7. With Tables $3,7,11,12,13$, and 14 it is straightforward to obtain the $\mathrm{CA}$ and $\mathrm{CC}$ when pass or fail decisions are made. The resulting classification table can be obtained by collapsing corresponding elements in the $\mathrm{CA} / \mathrm{CC}$ tables with five categories. Specifically, the sum of the elements of the first $2 \times 2$ block diagonal matrix and the

Table 14 Classification consistency by using the MPI method, long test

\begin{tabular}{llllll}
\hline & 1 & 2 & 3 & 4 & 5 \\
\hline 1 & .419 & 0.039 & 0.014 & 0.002 & 0.000 \\
2 & .043 & 0.029 & 0.030 & 0.006 & 0.000 \\
3 & .009 & 0.026 & 0.037 & 0.028 & 0.002 \\
4 & .001 & 0.008 & 0.021 & 0.092 & 0.027 \\
5 & .000 & 0.000 & 0.001 & 0.027 & 0.139 \\
$\lambda=.72$ & & & \multicolumn{4}{c}{ Chance corrected $=.48$} \\
\hline
\end{tabular}


Table 15 Error rates

\begin{tabular}{|c|c|c|c|c|c|c|}
\hline 12 Item & 1 & 2 & 3 & 4 & Unweighted & Weighted \\
\hline$P^{+}$ & .129 & 0.028 & 0.005 & 0.000 & .162 & .319 \\
\hline$P^{-}$ & .089 & 0.013 & 0.001 & 0.000 & .103 & .164 \\
\hline Sum & & & & & .265 & .483 \\
\hline \multicolumn{7}{|l|}{20 Item } \\
\hline$P^{+}$ & .103 & 0.012 & 0.000 & 0.000 & .115 & .164 \\
\hline$P^{-}$ & .091 & 0.007 & 0.000 & 0.000 & .098 & .128 \\
\hline Sum & & & & & .213 & .292 \\
\hline \multicolumn{7}{|l|}{40 Item } \\
\hline$P^{+}$ & 0.101 & 0.009 & 0.000 & 0.000 & .110 & .147 \\
\hline$P^{-}$ & 0.090 & 0.007 & 0.000 & 0.000 & .097 & .126 \\
\hline Sum & & & & & .207 & .273 \\
\hline
\end{tabular}

sum of the bottom $3 \times 3$ block diagonal matrix become elements along the diagonal of the new $\mathrm{CA} / \mathrm{CC}$ matrix. The trace is the new $\mathrm{CA} / \mathrm{CC}$ rate. Similarly, we can obtain the $\mathrm{CA} / \mathrm{CC}$ matrix if the binary cut is located elsewhere-for example, $\mathrm{c}_{1}, c_{3}$, or $c_{4}$. The summary statistics are provided in Table 16. Clearly, the $\mathrm{CA} / \mathrm{CC}$ rates are much higher when the decision is less complex: When there are five placement categories, the accuracy and consistency are in the $.60 \mathrm{~s}$ to $.70 \mathrm{~s}$; when there are only two categories, the numbers range typically in the upper .80 s or $.90 \mathrm{~s}$. This is very consistent with the observation that $\mathrm{CA}$ (and $\mathrm{CC}$ ) drops when the classification decisions concern more fine-grained categorization.

\section{Discussion}

In this article, we examined four item selection methods in terms of CA and CC. Several important findings are gleaned from this study. First, even though essentially all four item selection methods yield unbiased latent trait estimates with a rather short test, the variability of the latent trait estimate

Table 16 Classification accuracy and consistency at different cut locations

\begin{tabular}{lllll}
\hline 12 item & $c_{1}$ & $c_{2}$ & $c_{3}$ & $c_{4}$ \\
\hline$\gamma$ & .90 & 0.91 & 0.92 & 0.95 \\
$\lambda$ & .86 & 0.87 & 0.89 & 0.92 \\
20 item & & & & \\
$\gamma$ & .92 & 0.94 & 0.95 & 0.96 \\
$\lambda$ & .88 & 0.89 & 0.93 & 0.94 \\
40 item & & & & \\
$\gamma$ & .93 & 0.94 & 0.95 & 0.96 \\
$\lambda$ & .89 & 0.90 & 0.93 & 0.94 \\
\hline
\end{tabular}

plays an essential role in the CA and CC. A larger variability usually results in lower CA and CC.

Linking the standard error $(S E)$ of latent trait estimate to $\mathrm{CA}$ and $\mathrm{CC}$ has important implications. Bias and $S E$ (or $M S E$ ) are usually examined, but it is the CA and CC that are the ultimate outcomes. Setting a standard on $S E$ may not be as intuitive and as easily explained to the general public as setting up a standard on the CA and CC rates.

Second, given a test as short as 12 items, the MPI method does reasonably well at maintaining measurement precision and CA. Among the item selection methods investigated here, it is also the only item selection method that manages to produce parallel forms and, consequently, the only method to which the definition of $\mathrm{CC}$ holds. It also leads to a small test overlap rate, meaning that only a small number of common items appear on two independently administered adaptive tests. Thus, it makes the test less prone to test security breach. Therefore, the MPI method is recommended here. A future study comparing the performance of the MPI method and the mathematical programming approaches, such as the shadow test method, is warranted.

Moreover, the number of decision categories heavily influences $\mathrm{CA}$ and $\mathrm{CC}$. Test length will also impact CA and $\mathrm{CC}$. The positive effect of a longer test is more evident when more severe errors are accordingly weighted. However, the improvement gradually diminishes as the test gets longer and longer. As evidenced by results in this study, either there are only a small number of decision categories, or very long tests are needed, to achieve highly accurate and consistent classification decisions.

Results from this study have important practical implications. Simulation studies are particularly useful in providing information on $\mathrm{CA}$ and $\mathrm{CC}$, because in reality the true category that an examinee belongs to is never known. Therefore, before a testing program becomes operational or any major change is adopted to currently operational testing programs, it is beneficial to run a simulation study 
to investigate the $\mathrm{CA}$ and $\mathrm{CC}$ rates. In the context of linear testing and CACT, these indices are well discussed, but in the context of CAT, such discussion is sorely lacking. This study represents, to our knowledge, the first attempt in addressing both $\mathrm{CA}$ and $\mathrm{CC}$ in regular CAT. It shows how to compute $\mathrm{CA}$ and $\mathrm{CC}$ and eventually will help find out, given an item pool, a target examinee population, and an item selection method, how long the CAT needs to run to reach a certain level of $\mathrm{CA}$ and $\mathrm{CC}$.

Author note The authors would like to thank College Board for the support of this study.

\section{References}

Barrada, J. R., Olea, J., Ponsoda, V., \& Abad, F. J. (2010). A Method for the Comparison of Item Selection Rules in Computerized Adaptive Testing. Applied Psychological Measurement, 34, 438-452.

Buyske, S. (2005). Optimal design in educational testing. In M. P. F. Berger \& W. K. Wong (Eds.), Applied optimal designs (pp. 1-19). West Sussex, England: John Wiley \& Sons.

Chang, H., Qian, J., \& Ying, Z. (2001). $a$-stratified multistage CAT with b-blocking. Applied Psychological Measurement, 25, 333-341.

Chang, H., \& Ying, Z. (1996). A global information approach to computerized adaptive testing. Applied Psychological Measurement, 20, 213-229.

Chang, H., \& Ying, Z. (1999). $a$-stratified multistage computerized adaptive testing. Applied Psychological Measurement, 23, 211222.

Chen, S., Ankenmann, R. D., \& Spray, J. A. (2003). The relationship between item exposure and test overlap in computerized adaptive testing. Journal of Educational Measurement, 40, 129-145.

Cheng, Y., \& Chang, H. (2009). The maximum priority index method for severely constrained item selection in computerized adaptive testing. British Journal of Mathematical and Statistical Psychology, 62, 369-383.

Cheng, Y., Chang, H., Douglas, J., \& Guo, F. (2009). Constraintweighted a-stratification for computerized adaptive testing with non-statistical constraints: Balancing measurement efficiency and exposure control. Educational and Psychological Measurement, $69,35-49$.

Cohen, J. (1960). A coefficient of agreement for nominal scales. Educational and Psychological Measurement, 20, 37-46.

Cronbach, L. J., \& Gleser, G. C. (1957). Psychological tests and personnel decisions. Urbana, IL: University of Illinois Press.

Davey, T., \& Parshall, C. G. (1995, April). New algorithms for item selection and exposure control with computerized adaptive testing. Paper presented at the Annual Meeting of the American Educational Researsch Association, San Francisco, CA.

Eggen, T. J., \& Straetmans, G. J. J. M. (2000). Computerized adaptive testing for classifying examinees into three categories. Educational and Psychological Measurement, 60, 713-734.

Gnambs, T., \& Batinic, B. (2011). Polytomous adaptive classification testing: Effects of item pool size, test termination criterion and number of cutscores. Educational and Psychological Measurement, 71, 1006-1022.
Georgiadou, E., Triantafillou, E., \& Economides, A. (2007). A review of item exposure control strategies for computerized adaptive testing developed from 1983 to 2005. Journal of Technology, Learning, and Assessment, 5. Retrieved June 24, 2007 from http://www.jtla.org

Hambleton, R., \& Swaminathan, H. (1985). Item response theory: Principles and applications. Boston, MA: Kluwer-Nijhoff Publishing.

Kim, D., Choi, S. W., Um, K. R., \& Kim, J. (2006) A Comparison of Methods for Estimating Classification Consistency. Paper presented at the annual meeting of the National Council of Measurement in Education, San Francisco, CA.

Lee, W. (2010). Classification consitency and accuracy for complex assessments using item response theory. Journal of Educatinoal Measurement, 47, 1-17.

Lee, W., Hanson, B., \& Brennan, R. (2002). Estimating consistency and accuracy indices for multiple classifications. Applied Psychological Measurement, 26, 412-432.

Leung, C. K., Chang, H. H., \& Hau, K. T. (2005). Computerized adaptive testing: A mixture item selection approach for constrained situations. British Journal of Mathematical and Statistical Psychology, 58, 239-257.

Lord, F. M. (1980). Applications of item response theory to practical testing problems. Mahwah, NJ: Erlbaum.

Lunz, M. E., \& Stahl, J.A. (1998). Patterns of item exposure using a randomized CAT algorithm. Paper presented at the annual meeting of the National Council on Measurement in Education, San Diego, CA.

Moyer, E. L., Galindo, J. L., \& Dodd, B. G. (2012). Balancing Flexible Constraints and Measurement Precision in Computerized Adaptive Testing. Educational and Psychological Measurement. doi:10.1177/0013164411431838

Revuelta, J., \& Ponsoda, V. (1998). A comparison of item exposure control methods in computerized adaptive testing. Journal of Educational Measurement, 35, 311-327.

Rudner, L. M. (2005). Expected classification accuracy. Practical Assessment, Research \& Evaluation, 10(13). Available at http:// pareonline.net/getvn. asp? $\mathrm{v}=10 \& \mathrm{n}=13$

Rudner, L. M., \& Guo, F. (2011). Computer Adaptive Testing for Small Scale Programs and Instructional Systems. GMAC Research Reports, RR-11-01. Retrievable at http://www.gmac.com/NR/ rdonlyres/91495C8D-0276-41FA-93BE-6595979B8D24/0/ RR1101 $C$ ATforSmallScalePrograms.pdf

Spray, J. A., \& Reckase, M. D. (1994). Comparison of SPRT and sequntial Bayes procedures for classifying examinees into two categories using a computerized test. Journal of Educational and Behavioral Statistics, 21, 405-414.

Stocking, M. L., \& Swanson, L. (1993). A method for severely constrained item selection in adaptive testing. Applied Psychological Measurement, 17, 277-292.

Sympson, J., \& Hetter, R. (1985). Controlling item exposure rates in computerized adaptive testing. Proceedings of teh 27th annual meeting of the Militray Testing Association (pp. 973-977), San Diego, CA: Navy Personnel Research and Development Center.

Thompson, N. A. (2007). A practitioner's guide for variable-length computerized classification testing. Practical Assessment Research and Evaluation, 12. Available online: http://pareonline. net/getvn.asp? $\mathrm{v}=12 \& \mathrm{n}=1$

van der Linden, W. J. (2000). Constrained adaptive testing with shadow tests. In W. J. van der Linden \& C. A. W. Glas (Eds.), Computerized adaptive testing: Theory and practice (pp. 27-52). Boston, MA: Kluwer.

van der Linden, W. J. (2002). Constrained adaptive testing with shadow tests. In W. J. van der Linden \& C. A. W. Glas (Eds.), 
Computerized adaptive testing: Theory and practice (pp. 27-52). Boston: Kluwer-Nijhoff.

Wainer, H., et al. (2000). Computerized adaptive testing: A primer. Mahwah, New Jersey: Lawrence Erlbaum Associates.

Way, W. D. (1998). Protecting the integrity of computerized testing item pools. Educational Measurement: Issues and Practice, 17, $17-27$.
Weissman, A. (2007). Mutual information item selection in adaptive classification testing. Educational and Psychological Measurement, 67, 41-58.

Yang, X., Poggio, J. C., \& Glasnapp, D. R. (2006). Effects of estimation bias on multiple-category classification with an IRT-based adaptive classification procedure. Educational and Psychological Measurement, 66, 545-564. 